\title{
Influence of EMS-physician presence on survival after out-of-hospital cardiopulmonary resuscitation: systematic review and meta-analysis
}

\author{
Bernd W. Böttiger ${ }^{1 *}$, Michael Bernhard ${ }^{2}$, Jürgen Knapp ${ }^{3}$ and Peter Nagele ${ }^{4} \mathbb{D}$
}

\begin{abstract}
Background: Evidence suggests that EMS-physician-guided cardiopulmonary resuscitation (CPR) in out-of-hospital cardiac arrest (OOHCA) may be associated with improved outcomes, yet randomized controlled trials are not available. The goal of this meta-analysis was to determine the association between EMS-physician- versus paramedic-guided CPR and survival after OOHCA.

Methods and Results: Studies that compared EMS-physician- versus paramedic-guided CPR in OOHCA published until June 2014 were systematically searched in MEDLINE, EMBASE and Cochrane databases. All studies were required to contain survival data. Data on study characteristics, methods, and as well as survival outcomes were extracted. A random-effects model was used for the meta-analysis due to a high degree of heterogeneity among the studies $\left(P^{2}=44 \%\right)$. Return of spontaneous circulation [ROSC], survival to hospital admission, and survival to hospital discharge were the outcome measures.

Out of 3,385 potentially eligible studies, 14 met the inclusion criteria. In the pooled analysis $(n=126,829)$,

EMS-physician-guided CPR was associated with significantly improved outcomes compared to paramedic-guided

CPR: ROSC $36.2 \%$ (95 \% confidence interval [Cl] 31.0 - $41.7 \%$ ) vs. $23.4 \%$ (95 \% Cl 18.5 - $29.2 \%$ ) (pooled odds ratio [OR] 1.89, $95 \% \mathrm{Cl} 1.36-2.63, \mathrm{p}<0.001$ ); survival to hospital admission $30.1 \%$ (95 \% Cl $24.2-36.7 \%$ ) vs. $19.2 \%$ (95 \% Cl $12.7-28.1 \%$ ) (pooled OR 1.78, $95 \% \mathrm{Cl} 0.97-3.28, \mathrm{p}=0.06$ ); and survival to discharge $15.1 \%$ (95\% Cl 14.6 - $15.7 \%$ ) vs. $8.4 \%$ (95 \% Cl $8.2-8.5 \%$ ) (pooled OR 2.03, $95 \%$ Cl $1.48-2.79, \mathrm{p}<0.001)$.
\end{abstract}

Conclusions: This systematic review suggests that EMS-physician-guided CPR in out-of-hospital cardiac arrest is associated with improved survival outcomes.

Keywords: Cardiac arrest, Cardiopulmonary resuscitation, Outcomes, Emergency medical service physicians, Paramedics

\section{Background}

The optimal emergency medical service (EMS) system configuration and staffing for out-of-hospital cardiopulmonary resuscitation (CPR) are controversial [1-3]. In several countries, EMS physicians are an integral part of prehospital EMS teams and are often dispatched to the most severe cases, including cardiac arrest. EMS physicians have undergone special training in emergency medicine that often goes beyond current advanced cardiac

\footnotetext{
*Correspondence: bernd.boettiger@uk-koeln.de

${ }^{1}$ Department of Anaesthesiology and Intensive Care Medicine University

Hospital of Cologne, Kerpener Str. 62, 50937 Cologne, Germany

Full list of author information is available at the end of the article
}

life support standards [1-7]. Despite the intuitive appeal of having EMS physicians guiding out-of-hospital CPR, there is only limited evidence about the influence of EMSphysician-guided CPR on outcomes after out-of-hospital cardiac arrest (OOHCA). Studies comparing the effect of different EMS systems (i.e., EMS-physician-staffed versus nonphysician (paramedic)-staffed systems) and their effects on survival in OOHCA patients are notoriously difficult to conduct and thus are limited [1-3]. Interestingly, almost all large-scale comparative studies demonstrate a survival benefit associated with EMS-physician-guided CPR for OOHCA $[2-5,7]$. 
The goal of this study was therefore to summarize the existing evidence comparing EMS-physicianguided versus paramedic-guided $\mathrm{CPR}$ and survival after OOHCA.

\section{Methods}

The Preferred Reporting Items for Systematic reviews and Meta-Analyses (PRISMA) [8] and Meta-analysis Of Observational Studies in Epidemiology (MOOSE) guidelines [9] were followed in this meta-analysis.

\section{Search strategy}

We performed a literature search accessing MEDLINE, EMBASE, and Cochrane databases for studies published until June 2014 using the following search terms and keywords: PubMed: (Heart arrest [mh] OR ((cardiac [tw] OR heart [tw]) AND arrest [tw])) AND (prehospital [tw] OR pre-hospital [tw] OR out-of-hospital [tw] OR "emerg* physician" " [tw] OR "prehosp* physician" [tw]) AND (ALS [tw] OR advanced card* support* [tw] OR advanced cardiac life support [mh] OR resuscitat" [tw] OR resuscitation [mh] OR cardiopulmonary resuscitation [mh]). The search strategy was based on combinations of Medical Subject Heading terms and text words and was not restricted to a specific language or year of publication. Electronic databases were searched-Cochrane Database for Systematic Reviews and Central Register of Controlled Trials (http://www.cochrane.org/), MEDLINE (http://www. ncbi.nlm.nih.gov/PubMed), and EMBASE (https://www. elsevier.com/solutions/embase-biomedical-research) —and hand searches of journals, review articles, and books were performed. In addition, we manually checked the reference list of each article. The main focus of this study was on prospective clinical trials, and we also included analysis of retrospective observational cohort studies.

\section{Study selection}

Since no randomized controlled clinical trials were available, we included in this meta-analysis all prospective and retrospective observational cohort studies. The following eligibility criteria were required for inclusion: observational cohort studies; comparison between EMS-physicianguided and paramedic-guided CPR; survival data available; adult population; and OOHCA. Articles were considered if published in English or German. For the study by Hagihara et al. [10], we selected only the propensitymatched cohort to reduce selection bias $(n=9231$ EMSphysician-treated cardiac arrests versus 9231 paramedictreated cardiac arrests).

\section{Data extraction}

Information about sample size, study design, and characteristics was extracted from the articles as well as the following data: patients treated by EMS physicians and paramedics, patients achieving return of spontaneous circulation (ROSC), surviving to hospital admission, and to hospital discharge, as well as 30-day survival. Survival to hospital discharge was the primary outcome variable. If survival to hospital discharge data were not available, we used ROSC and hospital admission as the primary outcomes. We used 30-day survival data if survival to discharge data were not available.

\section{Statistical analysis}

We performed the analysis with the Comprehensive MetaAnalysis software, version 2.2.064 (Biostat, Englewood, NJ, USA). Risk ratios and $95 \%$ confidence intervals (CIs) were (re)calculated for each study and pooled in both a fixedeffects model and a random effects model. The Comprehensive Meta-Analysis software uses the inverse variance method for weighing studies. However, other methods can be selected, such as Mantel-Haenszel. The results in our meta-analyses did not differ between each method. Heterogeneity among studies was formally assessed by the $Q$ and $I^{2}$ statistics. Publication bias was tested with the Egger's regression test.

\section{Results}

The literature search identified 3153 publications that met the search criteria. Detailed evaluation of abstracts and full articles resulted in 14 studies that met inclusion and exclusion criteria (Fig. 1, Table 1) [4, 5, 7, 10-20]. Quality of the included studies was variable and the heterogeneity was high $\left(I^{2}=44 \%\right)$. The funnel plot of included studies shows a small likelihood of publication bias (Additional file 1: Figure S1). The total pooled sample size was 126,829 cardiac arrest patients.

In the pooled analysis, EMS-physician-guided CPR was associated with significantly improved outcomes compared with paramedic-guided CPR. The pooled estimate for ROSC for EMS-physician-guided CPR was $36.2 \%$ (95\% CI $31.0-41.7 \%)$ and for paramedics was $23.4 \%$ (95 \% CI 18.5-29.2 \%) (pooled odds ratio (OR) 1.89, 95 \% CI 1.362.63, $p$ <0.001) (Fig. 2a; Additional file 1: Figure S2A). The pooled estimated survival-to-hospital admission rate for EMS-physician-guided CPR was $30.1 \%$ (95 \% CI 24.2$36.7 \%$ ) and for paramedics was $19.2 \%$ (95 \% CI $12.7-$ $28.1 \%$ ) (pooled OR 1.78, $95 \%$ CI $0.97-3.28, p=0.06$; Fig. 2b; Additional file 1: Figure S2B). The pooled estimated survival-to-hospital discharge rate for EMS-physicianguided CPR was $15.1 \%$ (95\% CI 14.6-15.7\%) and for paramedics was $8.4 \%$ (95\% CI 8.2-8.5\%) (pooled OR 2.03, 95 \% CI 1.48-2.79, $p<0.001$; Fig. 2c; Additional file 1: Figure S2C).

\section{Discussion}

The results of this meta-analysis show that CPR guided by EMS physicians is associated with improved 


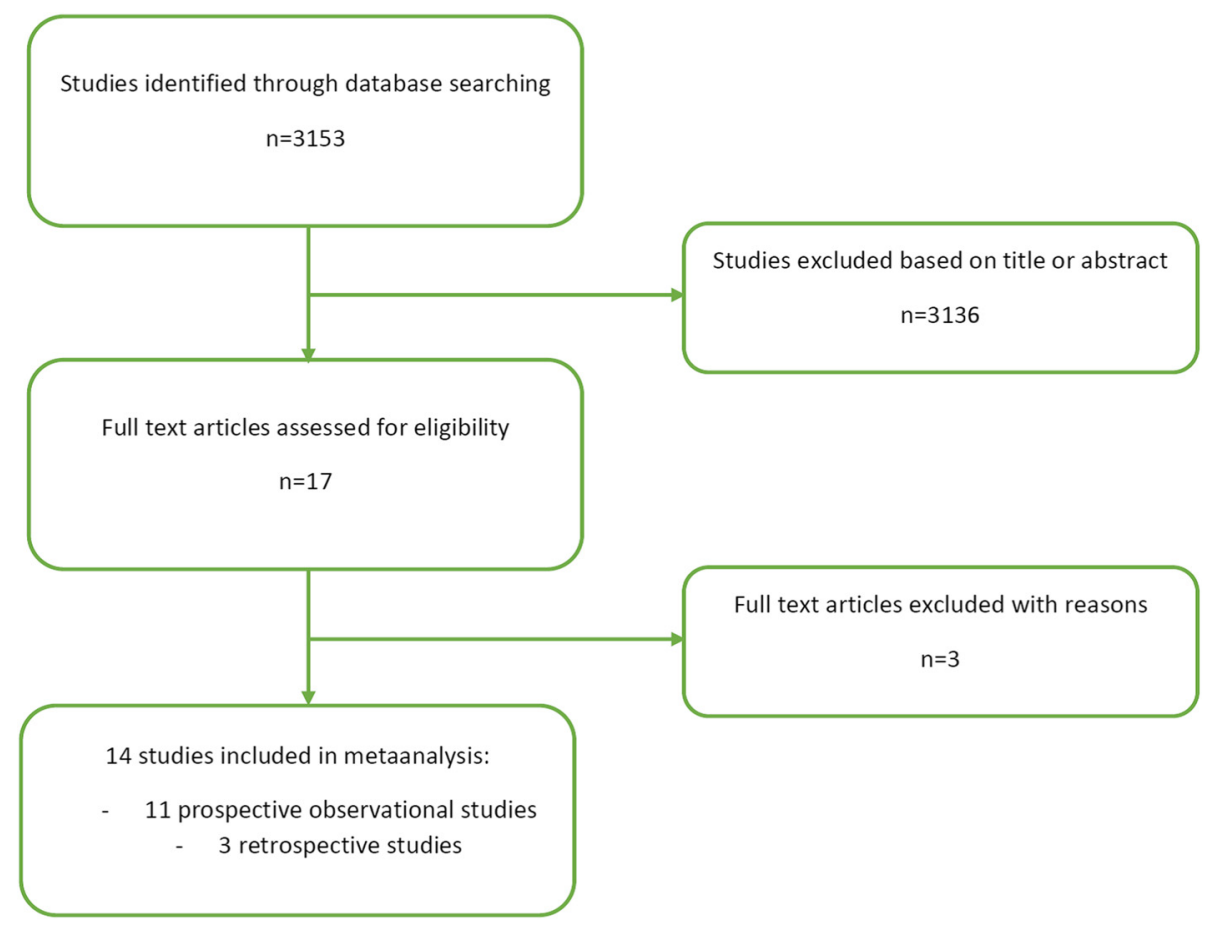

Fig. 1 Study selection process (based on PRISMA guidelines)

rates of ROSC, hospital admission, and hospital discharge compared with CPR guided by paramedics in OOHCA patients.

This meta-analysis included 14 international studies with a pooled sample size of more than 126,000 patients. Two studies from Japan [10, 17] accounted for nearly $90 \%$ of the total sample size and thus had the biggest weight in the meta-analysis. Because the individual studies were largely consistent in the effect size estimate, we did not perform sensitivity analyses excluding these two studies.

This study excluded several studies that had excellent methodology but did not directly compare EMSphysician-guided with paramedic-guided CPR, which may influence its generalizability. In several studies, EMS physicians provided advanced life support whereas paramedics were only allowed to perform basic life support without the administration of resuscitation drugs or advanced airway management. On the other hand, most countries that have a paramedic-only EMS system allow paramedics a nearly identical scope of prehospital practice compared with EMS physicians. Therefore, it is unclear whether our results show predominantly the superiority of advanced life support in OOHCA over basic life support or a true superiority of EMS-physician-guided CPR. In the multicenter Ontario Prehospital Advanced Life Support Study (OPLAS) study, Stiell et al. [21] directly compared advanced with basic life support for OOHCA and found no positive effect of advanced life support by paramedics on survival after OOHCA. This observation would argue against a predominant effect of advanced life support over basic life support.

This meta-analysis has several limitations. First, metaanalyses pool existing evidence and are thus dependent on the scientific quality of included studies. Typically, meta-analyses of randomized controlled trials provide the strongest and most robust evidence. In our study, no randomized controlled trials exist that compare EMSphysician-guided with paramedic-guided CPR and probably never will, due to the fact that whole states and countries operate one particular EMS system and switching systems is very costly. Despite the nonrandomized nature of studies included in this meta-analysis $[4,5,7,10-20]$, the evidence favoring EMS-physicianguided CPR for OOHCA appears to be robust since almost all studies found a similarly positive survival effect. Second, selection bias may have influenced individual study results. In some EMS systems, EMS-physicianstaffed ambulances may have not been dispatched to cases of OOHCA that were futile based on the assessment of an ambulance crew on the scene. Alternatively, EMS physicians may have determined on scene that initiation of CPR was not appropriate, which may have influenced the denominator of "potential cardiac arrests". This would have limited EMS-physician-guided CPR to OOHCA cases with a higher likelihood of successful resuscitation. 
Table 1 Characteristics of included studies with physicians and non-physicians (paramedics) in out-of-hospital CPR

\begin{tabular}{|c|c|c|c|c|c|c|c|c|c|c|c|c|}
\hline \multirow[t]{2}{*}{ Author } & \multirow[t]{2}{*}{ Design } & \multirow[t]{2}{*}{ Details } & \multicolumn{2}{|c|}{ Patients treated by } & \multicolumn{2}{|l|}{ ROSC } & \multicolumn{2}{|c|}{$\begin{array}{l}\text { Survival to hospital } \\
\text { admission }\end{array}$} & \multicolumn{2}{|c|}{$\begin{array}{l}\text { Survival to hospital } \\
\text { discharge }\end{array}$} & \multicolumn{2}{|l|}{ 30-day survival } \\
\hline & & & $\begin{array}{l}\text { Physician } \\
\text { (n) }\end{array}$ & $\begin{array}{l}\text { Paramedic } \\
(n)\end{array}$ & $\begin{array}{l}\text { Physician } \\
\text { (n/total, \%) }\end{array}$ & $\begin{array}{l}\text { Paramedic } \\
\text { (n/total, \%) }\end{array}$ & $\begin{array}{l}\text { Physician } \\
\text { (n/total, \%) }\end{array}$ & $\begin{array}{l}\text { Paramedic } \\
(n / \text { total, \%) }\end{array}$ & $\begin{array}{l}\text { Physician } \\
\text { (n/total, \%) }\end{array}$ & $\begin{array}{l}\text { Paramedic } \\
(n / \text { total, \%) }\end{array}$ & $\begin{array}{l}\text { Physician } \\
\text { (n/total, \%) }\end{array}$ & $\begin{array}{l}\text { Paramedic } \\
\text { (n/total, \%) }\end{array}$ \\
\hline $\begin{array}{l}\text { Olasveengen } \\
\text { et al., } 2009 \\
\text { [11] }\end{array}$ & $\begin{array}{l}\text { Retrospective } \\
\text { analysis of } \\
\text { registry data }\end{array}$ & $\begin{array}{l}\text { 2003-2008, } \\
\text { contemporaneous, } \\
\text { urban, same city, same } \\
\text { dispatch criteria }\end{array}$ & 232 & 741 & $\begin{array}{l}79 / 232 \\
34.0 \%\end{array}$ & $\begin{array}{l}242 / 741 \\
32.7 \%\end{array}$ & $\begin{array}{l}\text { With ROSC } \\
66 / 232 \text {, } \\
28.4 \% \text {; } \\
\text { with ongoing } \\
\text { CPR 22/232, } \\
9.5 \% \text {; all } \\
\text { hospital } \\
\text { 88/232, } 37.9 \%\end{array}$ & $\begin{array}{l}\text { With ROSC } \\
\text { 195/741, } \\
26.3 \% \text {; with } \\
\text { ongoing } \\
\text { CPR 98/741, } \\
13.2 \% \text {; all } \\
\text { hospital 293/ } \\
741,39.5 \%\end{array}$ & $\begin{array}{l}31 / 232 \\
13.4 \%\end{array}$ & $\begin{array}{l}\text { 78/741, } \\
10.5 \%\end{array}$ & $\begin{array}{l}\text { Not } \\
\text { reported }\end{array}$ & $\begin{array}{l}\text { Not } \\
\text { reported }\end{array}$ \\
\hline $\begin{array}{l}\text { Yen et al., } \\
2006 \text { [14] }\end{array}$ & $\begin{array}{l}\text { Prospective, } \\
\text { observational } \\
\text { multicenter } \\
\text { study }\end{array}$ & $\begin{array}{l}\text { 1999-2000, } \\
\text { contemporaneous, } \\
\text { urban, same city, } \\
\text { same dispatch criteria }\end{array}$ & 115 & 43 & $\begin{array}{l}\text { Not } \\
\text { reported }\end{array}$ & $\begin{array}{l}\text { Not } \\
\text { reported }\end{array}$ & $17 / 115,14.8 \%$ & $\begin{array}{l}16 / 43 \\
37.2 \%\end{array}$ & $\begin{array}{l}3 / 115 \\
2.6 \%\end{array}$ & $\begin{array}{l}4 / 43 \\
9.3 \%\end{array}$ & $\begin{array}{l}\text { Not } \\
\text { reported }\end{array}$ & $\begin{array}{l}\text { Not } \\
\text { reported }\end{array}$ \\
\hline $\begin{array}{l}\text { Oshige et al., } \\
2005 \text { [15] }\end{array}$ & $\begin{array}{l}\text { Prospective, } \\
\text { observational } \\
\text { study }\end{array}$ & $\begin{array}{l}\text { 2003, contemporaneous, } \\
\text { different urban and rural } \\
\text { areas: four areas with } \\
\text { physician-manned } \\
\text { ambulances compared } \\
\text { with four areas with } \\
\text { paramedic-staffed } \\
\text { ambulances, same } \\
\text { dispatch criteria }\end{array}$ & 120 & 222 & $\begin{array}{l}\text { Not } \\
\text { reported }\end{array}$ & $\begin{array}{l}\text { Not } \\
\text { reported }\end{array}$ & $49 / 120,40.8 \%$ & $\begin{array}{l}52 / 222 \\
23.4 \%\end{array}$ & $\begin{array}{l}\text { Not } \\
\text { reported }\end{array}$ & $\begin{array}{l}\text { Not } \\
\text { reported }\end{array}$ & $\begin{array}{l}13 / 120 \\
10.8 \%\end{array}$ & $\begin{array}{l}10 / 222 \\
4.5 \%\end{array}$ \\
\hline $\begin{array}{l}\text { Fischer et al., } \\
2003[4]\end{array}$ & $\begin{array}{l}\text { Prospective, } \\
\text { observational } \\
\text { study }\end{array}$ & $\begin{array}{l}\text { 1997, contemporaneous, } \\
\text { two different cities (city } \\
\text { of Bonn, Germany: } \\
\text { physician-manned } \\
\text { ambulance vs. city of } \\
\text { Birmingham, UK: } \\
\text { paramedic-staffed } \\
\text { ambulances) }\end{array}$ & 918 & 3380 & $\begin{array}{l}415 / 918 \\
45.2 \%\end{array}$ & $\begin{array}{l}554 / 3380 \\
16.4 \%\end{array}$ & $\begin{array}{l}371 / 918 \\
40.4 \%\end{array}$ & $\begin{array}{l}362 / 3380 \\
10.7 \%\end{array}$ & $\begin{array}{l}135 / 918 \\
14.7 \%\end{array}$ & $\begin{array}{l}135 / 3380 \\
4.0 \%\end{array}$ & $\begin{array}{l}\text { Not } \\
\text { reported }\end{array}$ & $\begin{array}{l}\text { Not } \\
\text { reported }\end{array}$ \\
\hline $\begin{array}{l}\text { Soo et al., } \\
1999[16]\end{array}$ & $\begin{array}{l}\text { Retrospective } \\
\text { observational } \\
\text { study }\end{array}$ & $\begin{array}{l}\text { 1991-1994, } \\
\text { contemporaneous, } \\
\text { same area }\end{array}$ & 70 & 551 & $\begin{array}{l}\text { Not } \\
\text { reported }\end{array}$ & $\begin{array}{l}\text { Not } \\
\text { reported }\end{array}$ & $\begin{array}{l}17 / 70 \\
24.3 \%\end{array}$ & $\begin{array}{l}86 / 551 \\
15.6 \%\end{array}$ & $\begin{array}{l}11 / 70 \\
15.7 \%\end{array}$ & $\begin{array}{l}32 / 551 \\
5.8 \%\end{array}$ & Not reported & $\begin{array}{l}\text { Not } \\
\text { reported }\end{array}$ \\
\hline $\begin{array}{l}\text { Kojima et al., } \\
2010 \text { [7] }\end{array}$ & $\begin{array}{l}\text { Prospective, } \\
\text { observational } \\
\text { study }\end{array}$ & $\begin{array}{l}\text { 2005-2008, } \\
\text { contemporaneous, } \\
\text { propensity } \\
\text { score-matched } \\
\text { analysis }\end{array}$ & 2072 & 2072 & $\begin{array}{l}555 / 2072 \\
26.8 \%\end{array}$ & $\begin{array}{l}249 / 2072 \\
12.0 \%\end{array}$ & $\begin{array}{l}\text { Not } \\
\text { reported }\end{array}$ & $\begin{array}{l}\text { Not } \\
\text { reported }\end{array}$ & $\begin{array}{l}\text { Not } \\
\text { reported }\end{array}$ & $\begin{array}{l}\text { Not } \\
\text { reported }\end{array}$ & $\begin{array}{l}336 / 2072 \\
16.2 \%\end{array}$ & $\begin{array}{l}227 / 2072 \\
11.0 \%\end{array}$ \\
\hline $\begin{array}{l}\text { Eisenburger } \\
\text { et al., } 2001 \\
\text { [12] }\end{array}$ & $\begin{array}{l}\text { Descriptive } \\
\text { observational } \\
\text { study with } \\
\text { prospective data }\end{array}$ & $\begin{array}{l}\text { 1991-1998, } \\
\text { contemporaneous, } \\
\text { same rural area }\end{array}$ & 105 & 13 & $\begin{array}{l}47 / 105 \\
44.8 \%\end{array}$ & $\begin{array}{l}7 / 13 \\
53.8 \%\end{array}$ & Not reported & $\begin{array}{l}\text { Not } \\
\text { reported }\end{array}$ & $\begin{array}{l}23 / 105 \\
21.9 \%\end{array}$ & $\begin{array}{l}3 / 13 \\
23.1 \%\end{array}$ & $\begin{array}{l}\text { 1-year } \\
\text { survival: 20/ } \\
\text { 105, } 19.0 \%\end{array}$ & $\begin{array}{l}\text { 1-year } \\
\text { survival: } \\
\text { 1/13, } 7.7 \%\end{array}$ \\
\hline
\end{tabular}


Table 1 Characteristics of included studies with physicians and non-physicians (paramedics) in out-of-hospital CPR (Continued)

\begin{tabular}{|c|c|c|c|c|c|c|c|c|c|c|c|c|}
\hline $\begin{array}{l}\text { Dickenson } \\
\text { et al., } \\
1997 \text { [13] }\end{array}$ & $\begin{array}{l}\text { Retrospective } \\
\text { case review }\end{array}$ & $\begin{array}{l}\text { 1994, } \\
\text { contemporaneous, } \\
\text { same suburban area }\end{array}$ & 9 & 40 & $\begin{array}{l}6 / 9 \\
66.7 \%\end{array}$ & $\begin{array}{l}12 / 40 \\
30.0 \%\end{array}$ & Not reported & $\begin{array}{l}\text { Not } \\
\text { reported }\end{array}$ & $\begin{array}{l}4 / 9 \\
44.4 \%\end{array}$ & $\begin{array}{l}2 / 40 \\
5.0 \%\end{array}$ & Not reported & Not reported \\
\hline $\begin{array}{l}\text { Hagihara } \\
\text { et al., } \\
2014 \text { [10] }\end{array}$ & $\begin{array}{l}\text { Prospective, } \\
\text { registry study }\end{array}$ & $\begin{array}{l}\text { 2005-2010, } \\
\text { contemporaneous, } \\
\text { nationwide in Japan, } \\
\text { physician not dispatched } \\
\text { to the scene but happened } \\
\text { to be present during rescue } \\
\text { mission for training of the } \\
\text { ambulance crew or } \\
\text { occasionally when the } \\
\text { patient collapsed }\end{array}$ & 9231 & 9231 & $\begin{array}{l}2774 / 9231 \\
30.1 \%\end{array}$ & $\begin{array}{l}1661 / 9231 \\
18.0 \%\end{array}$ & Not reported & $\begin{array}{l}\text { Not } \\
\text { reported }\end{array}$ & $\begin{array}{l}\text { Not } \\
\text { reported }\end{array}$ & $\begin{array}{l}\text { Not } \\
\text { reported }\end{array}$ & $\begin{array}{l}1441 / 9231 \\
15.6 \%\end{array}$ & $\begin{array}{l}1169 / 9231 \\
12.7 \%\end{array}$ \\
\hline $\begin{array}{l}\text { Yasunaga } \\
\text { et al., } \\
2010 \text { [17] }\end{array}$ & $\begin{array}{l}\text { Prospective, } \\
\text { registry study }\end{array}$ & $\begin{array}{l}\text { 2005-2007, } \\
\text { contemporaneous, } \\
\text { nationwide in Japan, } \\
\text { in several regions a } \\
\text { physician-staffed } \\
\text { ambulance is available }\end{array}$ & $\begin{array}{l}\text { without } \\
\text { BCPR } \\
1597 ; \\
\text { with } \\
\text { BCPR } \\
1916\end{array}$ & $\begin{array}{l}\text { without } \\
\text { BCPR } \\
53,482 \text {; } \\
\text { with BCPR } \\
38,077\end{array}$ & $\begin{array}{l}\text { Not } \\
\text { reported } \\
\text { not } \\
\text { reported }\end{array}$ & $\begin{array}{l}\text { Not } \\
\text { reported } \\
\text { not } \\
\text { reported }\end{array}$ & $\begin{array}{l}\text { Not reported } \\
\text { not reported }\end{array}$ & $\begin{array}{l}\text { Not } \\
\text { reported } \\
\text { not reported }\end{array}$ & $\begin{array}{l}\text { Not } \\
\text { reported } \\
\text { not } \\
\text { reported }\end{array}$ & $\begin{array}{l}\text { Not } \\
\text { reported } \\
\text { not } \\
\text { reported }\end{array}$ & $\begin{array}{l}\text { Without BCPR } \\
\text { 185/1597, } \\
11.6 \% \text {; with } \\
\text { BCPR 287/ } \\
\text { 1916, } 15.0 \% \text {; } \\
\text { all patients: } \\
\text { 472/3513, } \\
13.4 \%\end{array}$ & $\begin{array}{l}\text { Without BCPR } \\
3608 / 53,482 \text {, } \\
6.7 \% \text {; with } \\
\text { BCPR 3642/ } \\
\text { 38,077, 9.6\%; } \\
\text { all patients: } \\
7250 / 91559 \text {; } \\
7.9 \%\end{array}$ \\
\hline $\begin{array}{l}\text { Hampton } \\
\text { et al., } \\
1977 \text { [18] }\end{array}$ & $\begin{array}{l}\text { Prospective, } \\
\text { interventional } \\
\text { study }\end{array}$ & $\begin{array}{l}\text { probably 1975-1976, } \\
\text { contemporaneous, } \\
\text { same urban area }\end{array}$ & 19 & 46 & $\begin{array}{l}\text { Not } \\
\text { reported }\end{array}$ & $\begin{array}{l}\text { Not } \\
\text { reported }\end{array}$ & $9 / 19,47.4 \%$ & $8 / 46,17.4 \%$ & $\begin{array}{l}3 / 19 \\
15.8 \%\end{array}$ & $\begin{array}{l}2 / 46 \\
4.3 \%\end{array}$ & Not reported & Not reported \\
\hline $\begin{array}{l}\text { Mitchell } \\
\text { et al., } \\
1997 \text { [19] }\end{array}$ & $\begin{array}{l}\text { Prospective, } \\
\text { observational } \\
\text { study }\end{array}$ & $\begin{array}{l}\text { one calendar year in } \\
\text { the middle of the } \\
\text { nineties, contemporaneous, } \\
2 \text { different urban areas } \\
\text { (Edinburgh, UK: physician- } \\
\text { based vs. Milwaukee, USA: } \\
\text { paramedic-based) }\end{array}$ & 306 & 732 & $\begin{array}{l}116 / 306 \\
37.7 \%\end{array}$ & $\begin{array}{l}225 / 732 \\
31.1 \%\end{array}$ & 78/306, $25.5 \%$ & $\begin{array}{l}159 / 732 \\
21.7 \%\end{array}$ & $\begin{array}{l}38 / 306 \\
12.4 \%\end{array}$ & $\begin{array}{l}52 / 732 \\
7.2 \%\end{array}$ & Not reported & Not reported \\
\hline $\begin{array}{l}\text { Frandsen } \\
\text { et al., } \\
1991 \text { [20] }\end{array}$ & $\begin{array}{l}\text { Prospective, } \\
\text { interventional } \\
\text { study }\end{array}$ & $\begin{array}{l}\text { paramedic: } 1986-1989, \\
\text { physician: } 1988, \text { partly } \\
\text { contemporaneous, same } \\
\text { urban and rural area }\end{array}$ & 85 & 308 & $\begin{array}{l}\text { Not } \\
\text { reported }\end{array}$ & $\begin{array}{l}\text { Not } \\
\text { reported }\end{array}$ & $14 / 85,16.5 \%$ & $\begin{array}{l}31 / 308 \\
10.1 \%\end{array}$ & $\begin{array}{l}11 / 85 \\
12.9 \%\end{array}$ & $\begin{array}{l}10 / 308 \\
3.2 \%\end{array}$ & Not reported & Not reported \\
\hline $\begin{array}{l}\text { Fischer et al., } \\
2011 \text { [5] }\end{array}$ & $\begin{array}{l}\text { Prospective, } \\
\text { observational } \\
\text { study }\end{array}$ & $\begin{array}{l}\text { 2001-2004, physician-staffed: } \\
\text { urban (Bonn, Germany) and } \\
\text { rural (Cantabria, Spain), } \\
\text { paramedic-based: urban } \\
\text { (Coventry, UK and } \\
\text { Richmond, USA) }\end{array}$ & 263 & 833 & $\begin{array}{l}89 / 263 \\
33.8 \%\end{array}$ & $\begin{array}{l}214 / 833 \\
25.7 \%\end{array}$ & $84 / 263,31.9 \%$ & $\begin{array}{l}110 / 833 \\
13.2 \%\end{array}$ & $\begin{array}{l}\text { Not } \\
\text { reported }\end{array}$ & $\begin{array}{l}\text { Not } \\
\text { reported }\end{array}$ & Not reported & Not reported \\
\hline
\end{tabular}

CPR cardiopulmonary resuscitation, $R O S C$ return of spontaneous circulation, $B C P R$ bystander cardiopulmonary resuscitation 
a

Study name Outcome

$\begin{array}{llrrrrr} & & \begin{array}{r}\text { Odds } \\ \text { ratio }\end{array} & \begin{array}{c}\text { Lower } \\ \text { limit }\end{array} & \begin{array}{c}\text { Upper } \\ \text { limit }\end{array} & \text { Z-Value } & \text { p-Value } \\ \text { Olasveengen } & \text { ROSC } & 1.065 & 0.779 & 1.454 & 0.394 & 0.694 \\ \text { Fischer } & \text { ROSC } & 4.209 & 3.591 & 4.932 & 17.748 & 0.000 \\ \text { Kojima } & \text { ROSC } & 2.679 & 2.273 & 3.157 & 11.755 & 0.000 \\ \text { Eisenburger } & \text { ROSC } & 0.695 & 0.219 & 2.207 & -0.618 & 0.537 \\ \text { Dickenson } & \text { ROSC } & 4.667 & 0.998 & 21.814 & 1.958 & 0.050 \\ \text { Hagihara } & \text { ROSC } & 1.958 & 1.827 & 2.098 & 19.008 & 0.000 \\ \text { Mitchell } & \text { ROSC } & 1.376 & 1.041 & 1.819 & 2.239 & 0.025 \\ \text { Fischer 2 } & \text { ROSC } & 1.480 & 1.097 & 1.995 & 2.568 & 0.010 \\ & & 1.892 & 1.362 & 2.629 & 3.799 & 0.000\end{array}$

Odds ratio and $95 \% \mathrm{Cl}$

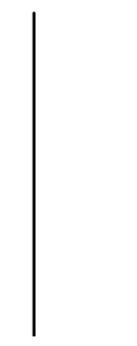

$0.01 \quad 0.1$

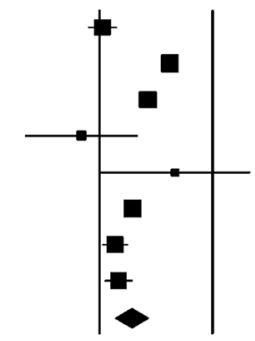

10100

Favours Paramedics Favours EMS-Physicians

b

\begin{tabular}{|c|c|c|c|c|c|c|c|c|c|c|}
\hline \multirow[t]{2}{*}{ Studyname } & \multirow[t]{2}{*}{ Outcome } & \multicolumn{5}{|c|}{ Statistics for each study } & & \multicolumn{3}{|c|}{ Odds ratio and $95 \% \mathrm{Cl}$} \\
\hline & & $\begin{array}{l}\text { Odds } \\
\text { ratio }\end{array}$ & $\begin{array}{l}\text { Lower } \\
\text { limit }\end{array}$ & $\begin{array}{c}\text { Upper } \\
\text { limit }\end{array}$ & Z-Value & p-Value & & & & \\
\hline Olasveengen & Surv. Admission & 0.934 & 0.690 & 1.266 & -0.438 & 0.661 & & & & \\
\hline Yen & Surv. Admission & 0.293 & 0.131 & 0.655 & -2.992 & 0.003 & & $\longrightarrow$ & & \\
\hline Oshige & Surv. Admission & 2256 & 1.398 & 3.641 & 3.333 & 0.001 & & & & \\
\hline Fischer & Surv. Admission & 5.655 & 4.765 & 6.709 & 19.850 & 0.000 & & & घ & \\
\hline Soo & Surv. Admission & 1.734 & 0.959 & 3.137 & 1.821 & 0.069 & & & & \\
\hline Hampton & Surv. Admission & 4.275 & 1.314 & 13.912 & 2.413 & 0.016 & & & 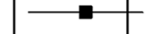 & \\
\hline Mitchell & Surv. Admission & 1.233 & 0.903 & 1.683 & 1.318 & 0.188 & & & & \\
\hline Frandsen & Surv. Admission & 1.762 & 0.890 & 3.488 & 1.626 & 0.104 & & & & \\
\hline \multirow[t]{4}{*}{ Fischer 2} & Surv. Admission & 3.084 & 2.222 & 4.281 & 6.735 & 0.000 & & & & \\
\hline & & 1.779 & 0.967 & 3.273 & 1.852 & 0.064 & & & & \\
\hline & & & & & & & 0.01 & 0.1 & 10 & 100 \\
\hline & & & & & & & \multicolumn{4}{|c|}{ Favours Paramedics Favours EMS-Physicians } \\
\hline
\end{tabular}

C

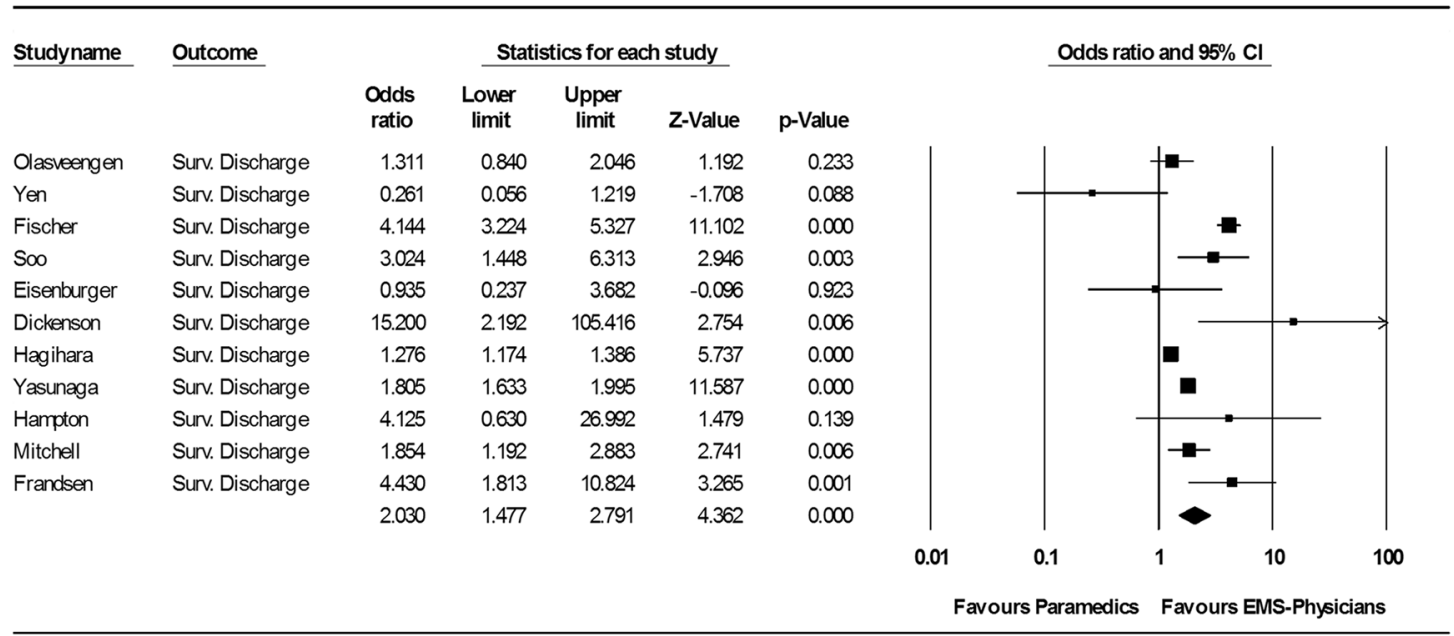

Fig. 2 Outcomes after CPR comparing EMS-physician-guided CPR with paramedic-guided CPR. a Return of spontaneous circulation (ROSC). b Survival to hospital admission. c Survival to hospital discharge. Cl confidence interval, EMS emergency medical services, Surv. survival 
Third, the geographic distribution of EMS systems is highly variable and is often influenced by many historical factors that all may have confounded the results of this meta-analysis.

If the results of this meta-analysis are true-that is, EMS-physician-guided CPR provides survival benefit in OOHCA over paramedic-guided CPR - what may be the causes? What could EMS physicians provide beyond what paramedics already contribute? First, it has been demonstrated that because of the limited number of invasive procedures performed by EMS crews (like airway management, tracheal intubation, etc.) in out-of-hospital patients, it is very difficult to obtain or maintain lifesaving skills [22-25]. As an example, even after 150 attempts at intubating the trachea in elective surgical patients under optimal conditions in the operating room the success rate is only $95 \%$ [26]. In the out-of-hospital setting, however, conditions are generally more difficult, leading to more challenging prehospital airway management $[27,28]$. On the other hand, EMS physicians are often anesthesiologists who maintain airway skills in the operating room while working only part-time in EMS medicine. Second, physician presence during CPR has been reported to increase compliance with guidelines, resulting in less hands-off time during CPR [11].

A randomized controlled trial comparing EMSphysician-guided versus paramedic-guided CPR will not be possible due to many reasons. Therefore, despite the significant limitations which are readily acknowledged, this systematic review provides the only available evidence for the effectiveness of a paramedic versus EMS-physicianbased emergency response system for prehospital cardiac arrest. Perhaps there may be opportunities for natural experiments when EMS systems change from paramedics to EMS physicians or vice versa. Additional analyses using large-scale registry data may help to elucidate this topic in the future.

\section{Conclusions}

In summary, findings from this meta-analysis suggest that CPR guided by EMS physicians is associated with improved survival compared with paramedic-guided CPR in OOHCA patients.

\section{Additional file}

Additional file 1: Figure S1 showing the funnel plot for publication bias analysis and Figure S2 showing the pooled event rates for ROSC, survival to hospital admission, and survival to hospital discharge. (DOCX $95 \mathrm{~kb}$ )

\section{Abbreviations}

Cl: Confidence interval; CPR: Cardiopulmonary resuscitation; EMS: Emergency medical service; MOOSE: Meta-analysis Of Observational Studies in Epidemiology; OOHCA: Out-of-hospital cardiac arrest; OR: Odds ratio;
PRISMA: Preferred Reporting Items for Systematic reviews and Meta-Analyses; ROSC: Return of spontaneous circulation.

\section{Competing interests}

All authors declare no conflicts of interest related to the topic of this manuscript.

\section{Authors' contributions}

BWB, MB, JK, and PN were responsible for the study design, acquisition of data, and drafting of the manuscript. PN was responsible for the statistical analysis. All authors contributed to the study concept, critical data interpretation, and the preparation and revision of the manuscript. All authors read and approved the final manuscript.

\section{Author details}

${ }^{1}$ Department of Anaesthesiology and Intensive Care Medicine University Hospital of Cologne, Kerpener Str. 62, 50937 Cologne, Germany. ${ }^{2}$ Emergency Department, University Hospital of Leipzig, Liebigstr. 20, 04103 Leipzig, Germany. ${ }^{3}$ Department of Anesthesiology and Pain Therapy, Bern University Hospital, Freiburgstr. 4, 3010 Bern, Switzerland. ${ }^{4}$ Department of

Anesthesiology, Washington University School of Medicine, St. Louis, MO, USA

Received: 29 April 2015 Accepted: 6 December 2015

Published online: 09 January 2016

\section{References}

1. Böttiger BW, Grabner C, Bauer H, Bode C, Weber T, Motsch J, et al. Long term outcome after out-of-hospital cardiac arrest with physician staffed emergency medical services: the Utstein style applied to a midsized urban/suburban area. Heart. 1999:82:674-9.

2. Bernhard M, Böttiger BW. Anaesthesiologists in emergency medicine: a win-win situation. Eur J Anaesthesiol. 2012;29:1-2.

3. Botker MT, Bakke SA, Christensen EF. A systematic review of controlled studies: do physicians increase survival with prehospital treatment? Scand J Trauma Resusc Emerg Med. 2009;17:12.

4. Fischer M, Krep H, Wierich D, Heister U, Hoeft A, Edwards S, et al. Comparison of the emergency medical services systems of Birmingham and Bonn: process efficacy and cost effectiveness. Anasthesiol Intensivmed Notfallmed Schmerzther. 2003:38:630-42.

5. Fischer M, Kamp J, Garcia-Castrillo Riesgo L, Robertson-Steel I, Overton J, Ziemann A, et al. Comparing emergency medical service systems-a project of the European Emergency Data (EED) Project. Resuscitation. 2011:82:285-93.

6. Bernhard M, Böttiger BW. Out-of-hospital endotracheal intubation of trauma patients: straight back and forward to the gold standard! Eur J Anaesthesiol. 2011;28:75-6.

7. Kojima SMK, Seino Y. A physician onboard the advanced life support unit has a clinical impact on outcome of witnessed patients with out-of-hospital cardiac arrest. Circulation. 2010;122:A108.

8. Moher D, Liberati A, Tetzlaff J, Altman DG, Group P. Preferred reporting items for systematic reviews and meta-analyses: the PRISMA statement. Ann Intern Med. 2009:151:264-9.

9. Stroup DF, Berlin JA, Morton SC, Olkin I, Williamson GD, Rennie D, et al. Meta-analysis of observational studies in epidemiology: a proposal for reporting. Meta-analysis Of Observational Studies in Epidemiology (MOOSE) group. JAMA. 2000:283:2008-12.

10. Hagihara A, Hasegawa M, Abe T, Nagata T, Nabeshima Y. Physician presence in an ambulance car is associated with increased survival in out-of-hospital cardiac arrest: a prospective cohort analysis. PLoS One. 2014;9:e84424.

11. Olasveengen TM, Lund-Kordahl I, Steen PA, Sunde K. Out-of hospital advanced life support with or without a physician: effects on quality of CPR and outcome. Resuscitation. 2009;80:1248-52.

12. Eisenburger $P$, Czappek $G$, Sterz F, Vergeiner G, Losert H, Holzer M, et al. Cardiac arrest patients in an alpine area during a six year period. Resuscitation. 2001:51:39-46.

13. Dickinson ET, Schneider RM, Verdile VP. The impact of prehospital physicians on out-of-hospital nonasystolic cardiac arrest. Prehosp Emerg Care. 1997;1:132-5. 
14. Yen ZS, Chen YT, Ko PC, Ma MH, Chen SC, Chen WJ, et al. Cost-effectiveness of different advanced life support providers for victims of out-of-hospital cardiac arrests. J Formos Med Assoc. 2006;105:1001-7.

15. Ohshige K, Shimazaki S, Hirasawa H, Nakamura M, Kin H, Fujii C, et al. Evaluation of out-of-hospital cardiopulmonary resuscitation with resuscitative drugs: a prospective comparative study in Japan. Resuscitation. 2005;66:53-61

16. Soo LH, Gray D, Young T, Huff N, Skene A, Hampton JR. Resuscitation from out-of-hospital cardiac arrest: is survival dependent on who is available at the scene? Heart. 1999:81:47-52.

17. Yasunaga $H$, Horiguchi $H$, Tanabe $S$, Akahane $M$, Ogawa $T$, Koike $S$, et al. Collaborative effects of bystander-initiated cardiopulmonary resuscitation and prehospital advanced cardiac life support by physicians on survival of out-of-hospital cardiac arrest: a nationwide population-based observational study. Crit Care. 2010;14:R199.

18. Hampton JR, Dowling M, Nicholas C. Comparison of results from a cardiac ambulance manned by medical or non-medical personnel. Lancet. 1977;1:526-9.

19. Mitchell RG, Brady W, Guly UM, Pirrallo RG, Robertson CE. Comparison of two emergency response systems and their effect on survival from out of hospital cardiac arrest. Resuscitation. 1997;35:225-9.

20. Frandsen F, Nielsen JR, Gram L, Larsen CF, Jorgensen HR, Hole P, et al. Evaluation of intensified prehospital treatment in out-of-hospital cardiac arrest: survival and cerebral prognosis. The Odense ambulance study. Cardiology. 1991;79:256-64.

21. Stiell IG, Wells GA, Field B, Spaite DW, Nesbitt LP, De Maio VJ, et al. Advanced cardiac life support in out-of-hospital cardiac arrest. N Engl J Med. 2004;351:647-56.

22. Gries A, Zink W, Bernhard M, Messelken M, Schlechtriemen T. Realistic assessment of the physician-staffed emergency services in Germany. Anaesthesist. 2006;55:1080-6.

23. Prause G, Wildner G, Kainz J, Bossner T, Gemes G, Dacar D, et al. Strategies for quality assessment of emergency helicopter rescue systems. The Graz model. Anaesthesist. 2007;56:461-5.

24. Deakin CD, Murphy D, Couzins M, Mason S. Does an advanced life support course give non-anaesthetists adequate skills to manage an airway? Resuscitation. 2010:81:539-43.

25. Sollid SJM, Sandberg M, Nakstad A, Bredmose P. Do anaesthesiologists in pre-hospital care need concomitant clinical practice? Scand J Trauma Resusc Emerg Med. 2013;21 Suppl 1:7.

26. Bernhard M, Mohr S, Weigand MA, Martin E, Walther A. Developing the skill of endotracheal intubation: implication for emergency medicine. Acta Anaesthesiol Scand. 2012;56:164-71.

27. Wang HE, Balasubramani GK, Cook LJ, Lave JR, Yealy DM. Out-of-hospital endotracheal intubation experience and patient outcomes. Ann Emerg Med. 2010:55:527-37.

28. von Goedecke A, Herff H, Paal P, Dorges V, Wenzel V. Field airway management disasters. Anesth Analg. 2007;104:481-3.

\section{Submit your next manuscript to BioMed Central and we will help you at every step:}

- We accept pre-submission inquiries

- Our selector tool helps you to find the most relevant journal

- We provide round the clock customer support

- Convenient online submission

- Thorough peer review

- Inclusion in PubMed and all major indexing services

- Maximum visibility for your research

Submit your manuscript at www.biomedcentral.com/submit

Biomed Central 